\title{
Riscos microbiológicos de fórmulas para lactentes
}

\author{
Microbiological risks of infant formulas
} Vivian Cristina da Cruz Rodrigues ${ }^{1 *}$ (D), Isabela Pereira de Oliveira ${ }^{1}$,
Rosângela Maria Neves Bezerra ${ }^{1}$, Adriane Elisabete Costa Antunes ${ }^{1}$

${ }^{1}$ Universidade Estadual de Campinas (UNICAMP), Faculdade de Ciências Aplicadas, Limeira/SP - Brasil

*Corresponding Author: Vivian Cristina da Cruz Rodrigues, Universidade Estadual de Campinas (UNICAMP), Faculdade de Ciências Aplicadas, Rua Pedro Zaccaria, 1300, Jardim Santa Luiza, CEP: 13484-350, Limeira/SP -

Brasil, e-mail: vivianrodrigues89@gmail.com

Cite as: Rodrigues, V. C. C., Oliveira, I. P., Bezerra, R. M. N., \& Antunes, A. E. C. (2019). Microbiological risks of infant formulas. Brazilian Journal of Food Technology, 22, e2018056. https://doi.org/10.1590/1981-6723.05618

\begin{abstract}
Resumo
O objetivo desse trabalho foi discorrer sobre os principais perigos biológicos encontrados em fórmulas infantis a partir dos relatos da literatura, especialmente de registros de surtos. Este estudo é de cunho exploratório por meio de revisão bibliográfica, sendo utilizados como fontes de dados sites de busca científica. Dentre os principais micro-organismos causadores de doenças ligadas à ingestão de fórmulas infantis estão o Cronobacter sakazakii e a Salmonella enterica, porém outras bactérias, como Clostridium botulinum, Klebsiella pneumoniae, Staphylococcus aureus e Bacillus cereus, podem ser responsáveis por contaminações destas fórmulas. Visto que lactentes apresentam os sistemas imunológico e metabólico ainda em desenvolvimento, estes representam um público mais vulnerável a contaminantes, fazendo-se fundamental o oferecimento de alimentos seguros desde o processamento na indústria até a administração nas residências e unidades hospitalares.
\end{abstract}

Palavras-chave: Fórmulas infantis; Contaminação de alimentos; Surtos; Patógeno; Salmonella; Cronobacter sakazakii.

\begin{abstract}
The objective of this paper was discuss the main biological hazards found in infant formulas from literature reports, especially from outbreak records. This study has an exploratory nature through a bibliographical review where scientific search sites were used as data sources. Cronobacter sakazakii and Salmonella enterica are among the main microorganisms that cause diseases associated with infant formulas ingestion; however, Clostridium botulinum, Klebsiella pneumoniae, Staphylococcus aureus and Bacillus cereus can also be responsible for infant formulas contaminations. Since infants have the immune and metabolic systems still in development, they represent a more vulnerable group to contaminants, making it essential to offer safe foods from processing in industry to administration in homes and hospital units.
\end{abstract}

Keywords: Infant formulas; Food contamination; Outbreaks; Pathogen; Salmonella; Cronobacter sakazakii. 


\section{Introdução}

A alimentação desempenha um papel essencial para a saúde humana desde o período gestacional. Os primeiros mil dias de vida da criança (270 dias da gestação e 730 dias até a criança completar dois anos de idade) são considerados uma janela de oportunidade para o desenvolvimento integral e adequado (Bhutta et al., 2008; Cunha et al., 2015). Durante essa fase, o crescimento acontece de forma rápida com a maturidade dos sistemas e órgãos, sendo este um período crítico pelo alto risco de infecções (Bula-Rudas et al., 2015; Mozetic et al., 2016).

O leite materno é o único alimento que o recém-nascido necessita até os seis meses de idade; no entanto, quando a amamentação não pode ser realizada, são necessários produtos apropriados para substituir nutricionalmente o leite humano, como as fórmulas infantis, capazes de proporcionar maior expectativa de vida aos lactentes (Sá et al., 2017). Portanto, a qualidade da alimentação oferecida nesse período é essencial para o completo desenvolvimento, influenciando na saúde e no bem-estar do indivíduo ao longo da vida (Cunha et al., 2015).

Do ponto de vista microbiológico, as fórmulas infantis podem sofrer contaminações, sendo relatados surtos e casos isolados de doenças vinculadas a seu consumo (Tabela 1). Em 2004, a Food and Agriculture Organization of the United Nations (FAO) e a World Health Organization (WHO) elaboraram um parecer avaliando os possíveis riscos microbiológicos em fórmulas infantis, tornando possível sua identificação e classificação (Food and Agriculture Organization of the United Nations, 2004). Os micro-organismos foram divididos em três categorias (A, B e C) mediante a comprovação científica do grau de causalidade de doenças (Tabela 2) (Food and Agriculture Organization of the United Nations, 2006). 
Tabela 1. Casos isolados e surtos por contaminação microbiológica de lactentes alimentados com fórmulas infantis.

\begin{tabular}{|c|c|c|c|c|c|}
\hline Ano/Local & Descrição & Alimento & Micro-organismo & Observação & Referência \\
\hline 1968-1987, Islândia & Infecção, 3 casos & Fórmula infantil & $\begin{array}{c}\text { E. sakazakii } \\
\text { (C. sakazakii) }\end{array}$ & 1 óbito & $\begin{array}{l}\text { Biering et al. } \\
\text { (1989) }\end{array}$ \\
\hline $\begin{array}{l}\text { 1988, Estados } \\
\text { Unidos }\end{array}$ & $\begin{array}{c}\text { Bacteremia, } \\
2 \text { casos; Infecção } \\
\text { urinária, } 1 \text { caso; } \\
\text { Diarreia } \\
\text { sanguinolenta, } \\
1 \text { caso }\end{array}$ & Fórmula infantil & $\begin{array}{l}\text { E. sakazakii } \\
\text { (C. sakazakii) }\end{array}$ & & $\begin{array}{l}\text { Simmons et al. } \\
\text { (1989) }\end{array}$ \\
\hline NI, Israel & $\begin{array}{c}\text { Infecção, } 5 \text { casos, } \\
\text { sendo } 3 \\
\text { assintomáticos }\end{array}$ & Fórmula infantil & $\begin{array}{c}\text { E. sakazakii } \\
\text { (C. sakazakii) }\end{array}$ & & Bar-Oz et al. (2001) \\
\hline 1998, Bélgica & $\begin{array}{c}\text { Enterocolite } \\
\text { necrosante, } \\
12 \text { casos }\end{array}$ & Fórmula infantil & C. sakazakii & 2 óbitos & $\begin{array}{c}\text { Van Acker et al. } \\
\text { (2001) }\end{array}$ \\
\hline $\begin{array}{c}\text { 2001, Estados } \\
\text { Unidos }\end{array}$ & Meningite, 10 casos & Fórmula infantil & $\begin{array}{c}\text { E. sakazakii } \\
\text { (C. sakazakii) }\end{array}$ & 1 óbito & $\begin{array}{l}\text { Centers for Disease } \\
\text { Control and } \\
\text { Prevention (2002) }\end{array}$ \\
\hline $\begin{array}{l}\text { 2002, Estados } \\
\text { Unidos }\end{array}$ & $\begin{array}{c}\text { Meningite, } 10 \text { casos } \\
\text { confirmados, } \\
2 \text { suspeitas e } \\
7 \text { "colonizados" }\end{array}$ & Fórmula infantil & $\begin{array}{l}\text { E. sakazakii } \\
\text { (C. sakazakii) }\end{array}$ & & Weir (2002) \\
\hline $\begin{array}{l}\text { 1966, Estados } \\
\text { Unidos }\end{array}$ & $\begin{array}{c}\text { Gastroenterite, } \\
2 \text { casos }\end{array}$ & $\begin{array}{l}\text { Leite em pó } \\
\text { desnatado }\end{array}$ & S. New Brunswick & $\begin{array}{l}\text { Equipamento } \\
\text { contaminado }\end{array}$ & Collins et al. (1968) \\
\hline 1985, Inglaterra & $\begin{array}{l}\text { Salmonelose, } \\
29 \text { casos }\end{array}$ & Fórmula infantil & S. ealing & $\begin{array}{l}1 \text { óbito. } \\
\text { Equipamento } \\
\text { contaminado }\end{array}$ & Rowe et al. (1987) \\
\hline $\begin{array}{l}\text { 1993, Canadá e } \\
\text { Estados Unidos }\end{array}$ & $\begin{array}{l}\text { Salmonelose, } \\
3 \text { casos }\end{array}$ & Fórmula infantil & $\begin{array}{l}S . \text { sorovar } \\
\text { Tennessee }\end{array}$ & $\begin{array}{l}\text { Equipamento } \\
\text { contaminado }\end{array}$ & $\begin{array}{l}\text { Centers for Disease } \\
\text { Control and } \\
\text { Prevention (1993) }\end{array}$ \\
\hline 1994, Espanha & $\begin{array}{l}\text { Salmonelose, } \\
48 \text { casos }\end{array}$ & Fórmula infantil & $\begin{array}{c}\text { S. enterica } \text { subsp. } \\
\text { enterica } \text { sorovar } \\
\text { Virchow }\end{array}$ & & Usera et al. (1996) \\
\hline $\begin{array}{l}\text { 1996-1997, Reino } \\
\text { Unido e França }\end{array}$ & $\begin{array}{l}\text { Salmonelose, } \\
17 \text { casos }\end{array}$ & Fórmula infantil & $\begin{array}{c}\text { S. enterica sorovar } \\
\text { Anatum }\end{array}$ & & $\begin{array}{c}\text { Threlfall et al. } \\
\text { (1998) }\end{array}$ \\
\hline 2001, Coréia & $\begin{array}{l}\text { Salmonelose, } \\
31 \text { casos }\end{array}$ & Fórmula infantil & $\begin{array}{l}\text { S. enterica sorovar } \\
\text { London }\end{array}$ & & Park et al. (2004) \\
\hline 2005, França & $\begin{array}{l}\text { Salmonelose, } \\
141 \text { casos }\end{array}$ & Fórmula infantil & $S$. Agona & & $\begin{array}{c}\text { Brouard et al. } \\
\text { (2007) }\end{array}$ \\
\hline 2008, França & $\begin{array}{c}\text { Salmonelose, } \\
3 \text { casos }\end{array}$ & Fórmula infantil & $\begin{array}{c}\text { S. enterica serotype } \\
\text { Give }\end{array}$ & & $\begin{array}{c}\text { Jourdan et al. } \\
\text { (2008) }\end{array}$ \\
\hline 2008, Espanha & $\begin{array}{l}\text { Salmonelose, } \\
23 \text { casos }\end{array}$ & Fórmula infantil & $\begin{array}{c}\text { S. enterica serotype } \\
\text { Kedougou }\end{array}$ & & Soler et al. (2008) \\
\hline 2010, Espanha & $\begin{array}{l}\text { Gastroenterite, } \\
289 \text { casos }\end{array}$ & Fórmula infantil & $\begin{array}{c}\text { S. enterica sorovar } \\
\text { Poona }\end{array}$ & $\begin{array}{l}84 \% \text { consumidores } \\
\text { da mesma fórmula. } \\
\text { Fórmula não } \\
\text { testada }\end{array}$ & Orvay et al. (2013) \\
\hline $\begin{array}{l}\text { 2017, França, } \\
\text { Espanha e Grécia }\end{array}$ & $\begin{array}{l}36 \text { casos e } \\
1 \text { suspeita }\end{array}$ & Fórmula infantil & $\begin{array}{c}\text { S. enterica sorovar } \\
\text { Agona }\end{array}$ & & Willsher (2018) \\
\hline 2001, Reino Unido & $\begin{array}{c}\text { Botulismo infantil, } \\
1 \text { caso }\end{array}$ & Fórmula infantil & C. botulinum tipo B & Não confirmado & Brett et al. (2005) \\
\hline NI, França & $\begin{array}{c}\text { Botulismo infantil, } \\
1 \text { caso }\end{array}$ & Fórmula infantil & C. botulinum & $\begin{array}{c}\text { Leite artificial e } \\
\text { materno não testado }\end{array}$ & $\begin{array}{c}\text { Bernardor et al. } \\
(2018)\end{array}$ \\
\hline
\end{tabular}

$\mathrm{NI}=$ não identificado. 
Tabela 2. Caracterização de micro-organismos ou toxinas de maior preocupação em fórmulas infantis em pó, com base na força de evidência de associação causal entre sua presença em fórmulas infantis em pó e doença em lactentes.

\begin{tabular}{|c|c|c|}
\hline Categoria & Causalidade & Bactérias \\
\hline $\mathrm{A}$ & Clara evidência & Salmonella enterica, Cronobacter sakazakii \\
\hline $\mathrm{B}$ & $\begin{array}{l}\text { Possível, porém sem comprovação científica } \\
\text { até o momento }\end{array}$ & $\begin{array}{l}\text { Escherichia vulneris, E. coli, Klebsiella pneumoniae, K. oxytoca, } \\
\text { Citrobacter koseri, C. freundii, Pantoea agglomerans, Enterobacter } \\
\text { cloacae, Hafnia alvei, Acinetobacter spp. e Serratia spp. }\end{array}$ \\
\hline $\mathrm{C}$ & Menos provável ou ainda não comprovada & $\begin{array}{l}\text { Clostridium botulinum, C. perfringens, C. difficile, Bacillus cereus, } \\
\text { Listeria monocytogenes, staphylococci coagulase-negativa e } \\
\text { Staphylococcus aureus }\end{array}$ \\
\hline
\end{tabular}

Fonte: Food and Agriculture Organization of the United Nations (2006).

Juntamente com a divulgação desse parecer, a World Health Organization enfatiza a necessidade de recomendações específicas para alimentos destinados a lactentes e crianças, e ressalta a importância da identificação de perigos e de um controle higiênico-sanitário eficiente para esses produtos (Food and Agriculture Organization of the United Nations, 2004). Diante do exposto, o objetivo deste trabalho foi apresentar um panorama sobre os principais perigos biológicos presentes em fórmulas infantis e como estes afetam a segurança das fórmulas e a saúde de lactentes e crianças na primeira infância.

\section{Metodologia}

O trabalho desenvolvido foi um estudo exploratório por meio de revisão bibliográfica, com base em artigos dos últimos 50 anos. O mecanismo de busca foi a consulta de sites, como Web of Science, Science Direct, Scielo, Google Acadêmico, arquivos disponíveis na Biblioteca Digital da UNICAMP e livros da Biblioteca da Universidade Estadual de Campinas. Os termos-chave para busca foram: Segurança dos Alimentos, Contaminação de Alimentos, Food safety, Contaminação de fórmulas infantis, Fórmulas infantis reconstituídas, Infant formula.

\section{Fórmulas infantis}

As composições das fórmulas infantis são específicas para a necessidade de cada lactente, considerando fatores como a faixa etária e a classificação do recém-nascido, a presença de alergias, a intolerância à lactose, o refluxo e as patologias, entre outros. Dentre todos os tipos de fórmulas, este artigo irá tratar especificamente das fórmulas destinadas a recém-nascidos, lactentes e crianças de primeira infância, sendo denominadas "de partida", quando indicadas para recém-nascidos a termo e com idades de até cinco meses e 29 dias; fórmulas para lactentes (desde que fortificadas com ferro) destinadas às idades entre 6 e 12 meses, e leites de transição, sendo estes equivalentes às fórmulas para lactentes, porém com teores mais elevados de cálcio, ferro e fósforo, podendo ser consumidos também por crianças de 12 a 36 meses (Sá et al., 2017).

De acordo com a Agência Nacional de Vigilância Sanitária (ANVISA), uma série de regras deve ser adotada para a produção de fórmulas infantis adequadas (Brasil, 2014). As regulamentações abrangem desde os ingredientes a serem utilizados na produção até a comercialização dos produtos, sendo estas as RDC n. ${ }^{\circ}$ 42/2011, RDC n. ${ }^{\circ}$ 46/2011, RDC n. ${ }^{\circ}$ 43/2011, RDC n. ${ }^{\circ}$ 44/2011, e RDC n. ${ }^{\circ}$ 45/2011, e a Lei n. ${ }^{\circ} 11.265 / 2006$. Além das RDCs que regularizam as fórmulas infantis, esses produtos precisam seguir critérios microbiológicos específicos, como os descritos na RDC 12/2001, que expõe tecnicamente os padrões microbiológicos de alimentos (Tabela 3) (Brasil, 2001), e no Codex 066/2008, que trata dos critérios microbiológicos exclusivos das fórmulas infantis (Tabelas 4 e 5) (Food and Agriculture Organization of the United Nations, 2009). 
Tabela 3. Critério microbiológico para alimentos infantis, incluindo fórmulas.

\begin{tabular}{|cccccc}
\hline Micro-organismos & TI & \multicolumn{3}{c}{ TR } \\
\cline { 3 - 7 } & & $\mathbf{n}$ & c & m & M \\
\hline Coliformes a $35^{\circ} \mathrm{C} / \mathrm{g}(\mathrm{mL})$ & 10 & 5 & 2 & $<3$ & 10 \\
Coliformes a $45^{\circ} \mathrm{C} / \mathrm{g}$ & Aus & 5 & 0 & Aus & - \\
Estaf. Coag. fórmulas $/ \mathrm{g}(\mathrm{mL})$ & Aus & 5 & 0 & Aus & - \\
B. cereus $/ \mathrm{g}(\mathrm{mL})$ & $10^{2}$ & 5 & 1 & 10 & $10^{2}$ \\
Salmonella $\mathrm{sp} / 25 \mathrm{~g}(\mathrm{~mL})$ & Aus & 10 & 0 & Aus & - \\
\hline
\end{tabular}

Fonte: RDC 12/2001 (Brasil, 2001). TI = Tolerância para amostra indicativa. $\mathrm{TR}=$ Tolerância para amostra representativa. $\mathrm{n}=$ número de unidades colhidas aleatoriamente de um mesmo lote e analisadas individualmente; $\mathrm{c}=$ número máximo aceitável de amostras com contagens entre os limites de $\mathrm{m}$ e M (plano de três classes). $\mathrm{m}$ = limite que, em um plano de três classes, separa o lote aceitável do produto ou lote com qualidade intermediária aceitável. $\mathrm{M}=$ limite que, em plano de duas classes, separa o produto aceitável do inaceitável. Em um plano de três classes, M separa o lote com qualidade intermediária aceitável do lote inaceitável. Valores acima de M são inaceitáveis. Aus = Ausência, em que c é igual a zero, aplica-se o plano de duas classes.

Tabela 4. Critério microbiológico para fórmulas “de partida”, fórmula para lactentes com necessidades dietoterápicas específicas e para leite humano fortificado.

\begin{tabular}{lccccc}
\hline \multicolumn{1}{c}{ Micro-organismos } & n & c & m & M & Plano de Classes \\
\hline Cronobacter sakazakii e outras C. Espécies & 30 & 0 & $0 / 10 \mathrm{~g}$ & - & 2 \\
Salmonella & 60 & 0 & $0 / 25 \mathrm{~g}$ & - & 2 \\
Bactérias anaeróbias mesófilas (UFC) & 5 & 2 & $500 / \mathrm{g}$ & $5000 / \mathrm{g}$ & 3 \\
\multicolumn{1}{c}{ Enterobacteriaceae } & 10 & 2 & $0 / 10 \mathrm{~g}$ & $\mathrm{NA}$ & 2 \\
\hline
\end{tabular}

Fonte: Codex 066/2008 (Food and Agriculture Organization of the United Nations, 2009). $\mathrm{n}=$ número de amostras que devem obedecer ao critério $\mathrm{c}$. $\mathrm{c}=$ número máximo de amostras contaminadas em um plano de 2 ou 3 classes. $\mathrm{m}=$ limite microbiológico para a amostra estar adequada em um plano de 2 classes. $\mathrm{M}$ = limite microbiológico para a amostra estar adequada em um plano de 3 classes. NA = não aplicável.

Tabela 5. Critério microbiológico para fórmulas destinadas a lactentes, fórmulas de transição e para crianças de primeira infância com necessidades dietoterápicas específicas.

\begin{tabular}{cccccc}
\hline Micro-organismos & n & c & m & M & Plano de Classes \\
\hline Salmonella & 60 & 0 & $0 / 25 \mathrm{~g}$ & - & 2 \\
Bactérias anaeróbias mesófilas (UFC) & 5 & 2 & $500 / \mathrm{g}$ & $5000 / \mathrm{g}$ & 3 \\
Enterobacteriaceae & 10 & 2 & $0 / 10 \mathrm{~g}$ & $\mathrm{NA}$ & 2 \\
\hline
\end{tabular}

Fonte: Codex 066/2008 (Food and Agriculture Organization of the United Nations, 2009). $\mathrm{n}=$ número de amostras que devem obedecer ao critério c. $\mathrm{c}=$ número máximo de amostras contaminadas em um plano de 2 ou 3 classes. $\mathrm{m}=$ limite microbiológico para a amostra adequada em um plano de 2 classes. $\mathrm{M}=$ limite microbiológico para a amostra adequada em um plano de 3 classes. NA = não aplicável.

Contaminações microbiológicas podem ocorrer durante a produção industrial (Figura 1), a estocagem ou até mesmo durante o preparo das fórmulas infantis, sendo necessárias orientações de condutas de assepsia nos ambientes de preparo e armazenamento após reconstituição (Food and Agriculture Organization of the United Nations, 2004, 2006). 


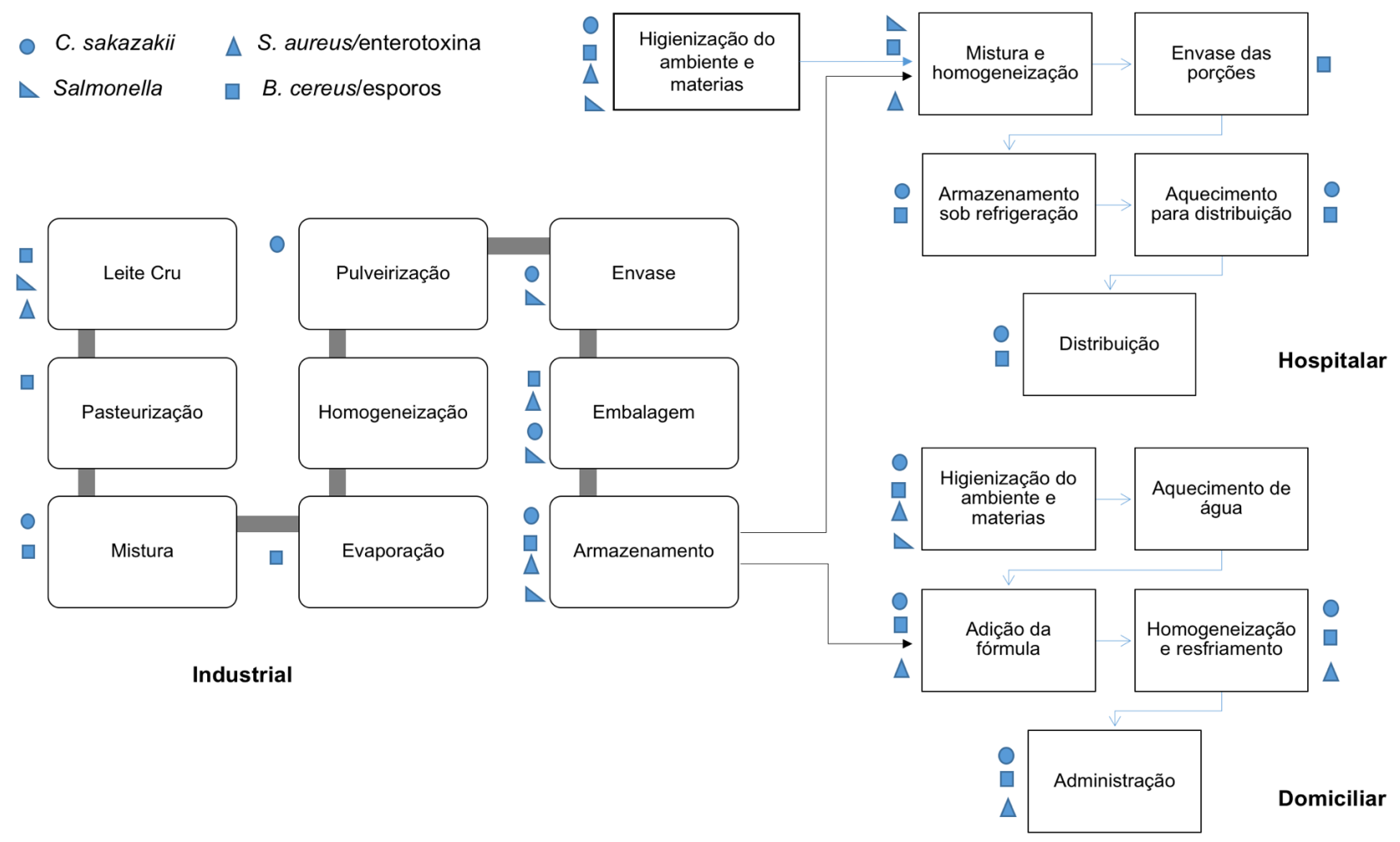

Figura 1. Fluxograma genérico da produção de fórmulas infantis e seu preparo em ambiente hospitalar e domiciliar. Fonte: Rossi (2007); World Health Organization (2007); Wu et al. (2018).

Os processos industrias de obtenção das fórmulas infantis apresentam etapa letal, geralmente constituindo-se em tratamento térmico, que permite a redução significativa ( 8 a 10 unidades de log ou mais) de micro-organismos em sua forma vegetativa (International Commision on Microbial Specifications for Foods, 2015). Desta forma, a presença de patógenos, tais como Salmonella e Cronobacter spp. em produtos acabados é possivelmente decorrente de contaminação pós-processo (International Commision on Microbial Specifications for Foods, 2015).

Para a reconstituição de fórmulas infantis em ambiente domiciliar ou em lactários, um fator essencial é a qualidade da água utilizada, recomendando-se que esta seja fervida e posteriormente resfriada até uma temperatura de aproximadamente $70^{\circ} \mathrm{C}$, garantindo eficiência na manutenção da segurança do produto e de seu valor nutricional (Food and Agriculture Organization of the United Nations, 2006). Outra etapa importante é garantir a correta higienização de todos os instrumentos e superfícies que serão utilizados durante a reconstituição, uma vez que a presença de resíduos pode causar recontaminação de utensílios e a formação de biofilmes, o que pode ocasionar contaminação cruzada de outros alimentos, pois micro-organismo patógenos e/ou seus esporos podem sobreviver longos períodos em alimentos com baixa atividade de água como as fórmulas infantis (World Health Organization, 2007; Beuchat et al., 2013).

\section{Contaminantes biológicos}

\subsection{Cronobacter sakazakii}

A Cronobacter sakazakii (C. sakazakii) é um patógeno oportunista emergente, sendo reconhecidamente um novo agente etiológico causador de doenças de origem alimentar (Machado, 2013). Dentre os riscos, pode-se citar meningite, enterocolite necrosante, sepse e bacteremia, alcançando taxas de mortalidade de até $80 \%$ e com possibilidade de sequela neurológica para os sobreviventes (Fiore et al., 2008). De acordo com a Food and Agriculture Organization of the United Nations (2004), a C. sakazakii é uma das bactérias 
classificadas na categoria A como veículo e fonte de infecção para lactentes e crianças, ou seja, a evidência de causalidade microbiológica e epidemiológica é cientificamente comprovada.

O processo de pasteurização consegue inativar a bactéria, no entanto pode haver contaminação cruzada de alimentos na planta de produção ou mesmo durante a reconstituição da fórmula, pois, quando presentes em uma superfície, estas são capazes de formar biofilme e produzir substâncias poliméricas extracelulares que a protegem do ambiente externo, impedindo sua eliminação (Lehner et al., 2005). O mecanismo de patogenicidade desse micro-organismo não é totalmente claro até o momento. Acredita-se que a menor acidez gástrica dos recém-nascidos contribua para a sobrevivência da bactéria, facilitando sua chegada e proliferação no intestino. Esta condição favorece a colonização das mucosas intestinais por patógenos e sua translocação para outros tecidos. Somada a isso, a síntese de diferentes compostos pode interferir na permeabilidade da barreira hematoencefálica, levando à infecção das meninges e do cérebro (Iversen \& Forsythe, 2003; Fiore et al., 2008).

Surtos e casos isolados associando a C. sakazakii com a transmissão de doenças podem ser encontrados na literatura, sendo alguns desses ocorridos no Brasil, como mostra a revisão de Brandão et al. (2018). Estudos apontam as fórmulas infantis como um dos principais veículos de doenças transmitidas por alimentos para crianças, principalmente menores de um ano de idade, prematuros e/ou com baixo peso (Food and Agriculture Organization of the United Nations, 2004; Fiore et al., 2008). Muytjens et al. (1988) analisaram 141 amostras de fórmulas infantis em pó de 35 países e constataram que 52,2\% estavam contaminadas com bactérias da família Enterobacteriaceae, sendo 14\% destas (13 países) identificadas como C. sakazakii. Trabalho semelhante foi realizado no Canadá por Nazarowec-White \& Farber (1997) e, como resultado, das 120 amostras analisadas, $6,7 \%$ estavam contaminadas.

Um surto de enterocolite necrosante atingiu 12 recém-nascidos na Bélgica em 1998, resultando em dois óbitos. Após estudo mais detalhado, observou-se que 10 dos 12 lactentes foram alimentados com a mesma marca de fórmula infantil em pó, sendo a C. sakazakii isolada do estômago, sangue e "swab" anal de seis deles, assim como das latas do mesmo lote de leite em pó (Van Acker et al., 2001). Estudos em diferentes países com amostras de fórmulas infantis obtidas de comércios varejistas ou online e de farmácias hospitalares, detectaram a presença de $C$. sakazakii em 5,3\% a 7,2\% do total das amostras analisadas, demonstrando que existe um risco constante de contaminação neste produto (Kim et al., 2011; Mardaneh \& Dallal, 2017).

Recentemente, foram publicados trabalhos que visavam analisar a possível presença de $C$. sakazakii em fórmulas infantis comercializadas na China. Fei et al. (2017) encontram contaminação de 2,8\% (56/2020) das amostras de fórmulas coletadas. Resultado semelhante foi observado por Xin et al. (2018), que constataram contaminação de $3,4 \%$ das 119 amostras que incluíam fórmulas infantis (88/119) e fórmulas infantis para lactentes de 6 a 12 meses (31/119). Outro estudo realizado por uma equipe chilena analisou 90 amostras de fórmulas infantis em pó e líquidas de quatro países (Chile, Estados Unidos, Holanda e Singapura) comercializadas em farmácias e supermercados no Chile. Das 90 amostras, 79 foram positivadas para micro-organismos aeróbios, sendo 55\% dessas bactérias identificadas como Enterobacteriaceae; Enterobacter cloacae; Enterobacter hormaechei, e Klebsiella pneumoniae. Além disso, do total avaliado, 8,8\% estava contaminado por C. sakazakii (Parra-Flores et al., 2018).

Este alerta vem despertando a necessidade de buscar maneiras rápidas e eficientes de identificar patógenos em alimentos infantis, visando reduzir os riscos de novos surtos e melhorando a saúde de uma população tão vulnerável como os lactentes. Neste sentido, Morlay e colaboradores, percebendo a importância da detecção de patógenos do leite como a C. sakazakii e a Salmonella spp., desenvolveram um método rápido (menos de um dia) e específico denominado Plasmonic Immuno-Assay (PlasmIA), que detecta baixos níveis de Cronobacter spp. e outros micro-organismos gram-negativos em fórmulas infantis (Morlay et al., 2016). 


\subsection{Salmonella enterica}

A presença de Salmonella enterica (S. enterica) em alimentos infantis é uma das principais responsáveis por infecções acometendo crianças menores de um ano de idade, podendo levar à morte lactentes imunodebilitados (Bula-Rudas et al., 2015). Essa bactéria se mostra responsável por quadros de diarreias, podendo evoluir para meningite ou bacteremia (Cahill et al., 2008). A transmissão pode ocorrer por falta de higiene, contaminação das matérias-primas, falhas no processamento ou mesmo contaminação ambiental após o tratamento térmico (Food and Agriculture Organization of the United Nations, 2004).

Após a ingestão do alimento contaminado, as bactérias sobreviventes ao ambiente ácido do estômago chegam ao intestino e iniciam seu processo de proliferação ao alcançarem a lâmina própria (Ohl \& Miller, 2001; BulaRudas et al., 2015). Comumente, esses micro-organismos agem localmente, sendo responsáveis por infecções gastrointestinais. Uma vez fagocitados por células de defesa, se proliferam e dão início a uma resposta inflamatória que estimula o aumento da secreção de água e eletrólitos, provocando diarreia (Haimovich \& Venkatesan, 2006; Shinohara et al., 2008). Além disso, podem migrar para outros órgãos, ampliando o risco de desenvolvimento de septicemia e morte dos infectados (Ohl \& Miller, 2001; Bula-Rudas et al., 2015).

Juntamente com a C. sakazakii, a Salmonella enterica é classificada na categoria A de perigos da Food and Agriculture Organization of the United Nations (2004). Em relação à Salmonella sp., para que um alimento seja considerado microbiologicamente seguro para consumo, sua presença deve ser negativada em 60 amostras de $25 \mathrm{~g}$ tomadas de um lote de fórmulas infantis de forma aleatória (Brasil, 2001). Assim como em outras doenças transmitidas por alimentos, a notificação é baixa por razões como a dificuldade de identificação de sorotipos e de inclusão dessas análises nas rotinas de serviços de saúde ou mesmo a transferência de responsabilidade da doença a outros fatores (Food and Agriculture Organization of the United Nations, 2006).

Diversos surtos relacionados com S. enterica e fórmulas infantis são registrados ao redor do mundo. Em 1994, três casos de contaminação hospitalar na Espanha causadas por uma cepa atípica de Salmonella sp. foram associados ao consumo de fórmula infantil (Usera et al., 1996). Na busca pela elucidação da origem e propagação dessa cepa, foi elaborado um programa de vigilância pelo National Epidemiology Center (NEC) que identificou 48 neonatos (menores de sete meses) que apresentaram síndrome gastroentérica aguda e confirmação positiva para Salmonella enterica subsp. enterica sorovar Virchow; além disso, houve a aplicação de questionários que incluíam perguntas, como contato com pessoas que tiveram diarreia, viagem para o exterior, alimentos consumidos e a marca da fórmula láctea utilizada. As fórmulas infantis em uso foram analisadas laboratorialmente durante aproximadamente seis meses, possibilitando a detecção da fonte de contaminação e a retirada do produto e dos lotes de circulação (Usera et al., 1996).

Um extenso estudo foi realizado por Yang et al. (2014) entre 2010 e 2012, na China, com 705 alimentos infantis à base de leite, dentre eles 246 fórmulas em pó obtidas de supermercados e lojas de varejo, sendo identificada a presença de $S$. enterica em 24 amostras, em que 5/246 (2\%) eram de leite em pó. No ano de 2017, um surto de Salmonella enterica sorovar Agona ligado a ingestão de fórmulas infantis de diversos segmentos foi registrado, atingindo pelo menos 37 crianças menores de seis meses de idade, sendo 35 na França, uma na Espanha e um caso de suspeita na Grécia. Mais de 12 milhões de caixas afetadas foram retiradas das prateleiras de supermercados de 83 países (Whitworth, 2017; Willsher, 2018). Em alguns casos, não é possível determinar qual foi a fonte de contaminação da fórmula e se ela ocorreu antes ou após a abertura da embalagem, sendo necessário, portanto, o cuidado no preparo e na higienização dos utensílios utilizados durante a reconstituição (Cahill et al., 2008). 


\subsection{Outros patógenos}

\subsubsection{Clostridium botulinum}

Micro-organismos do gênero Clostridium podem causar o distúrbio neuromuscular conhecido como botulismo (Rosow \& Strober, 2015). Existem vários tipos de botulismo, sendo um dos mais comuns o infantil, que atinge crianças menores de um ano de idade (especialmente até os seis meses) e pode ser causado principalmente pela espécie Clostridium botulinum (C. botulinum), sendo esta capaz de sintetizar sete neurotoxinas nomeadas de A a G (Brook, 2007; American Academy of Pediatrics, 2012; Rosow \& Strober, 2015). Atualmente, o botulismo infantil passou a ser denominado botulismo intestinal, visto que pode acometer também adultos em algumas condições que cursem com desequilíbrio da microbiota intestinal (tais como cirurgias intestinais, doença inflamatória intestinal e uso prolongado de antibióticos), bem como na presença de acloridria gástrica (Brook, 2007; American Academy of Pediatrics, 2012).

$\mathrm{O}$ botulismo infantil tem início com a ingestão de esporos que colonizam o trato gastrointestinal e produzem toxinas que se espalham pelo lúmen intestinal e são transportadas para a circulação, alcançando células colinérgicas, como as dos nervos periférico e craniano; as toxinas se ligam irreversivelmente a essas células, bloqueando a liberação de acetilcolina, o que pode causar paralisia simétrica descendente e hipotonia, e em casos mais severos, torna-se necessária a utilização de ventilação artificial (Brook, 2007; Rosow \& Strober, 2015). No caso dos bebês, a toxinfecção é facilitada devido ao processo de desenvolvimento da microbiota e do alto $\mathrm{pH}$ intestinal, condições nas quais os esporos desse micro-organismo podem germinar e produzir toxinas, principalmente neurotoxinas A e B (Bula-Rudas et al., 2015; Rosow \& Strober, 2015). Dentre as principais fontes de contaminação, está o mel, muitas vezes utilizado para adoçar as fórmulas infantis, sendo assim contraindicado para crianças menores de um ano de idade (American Academy of Pediatrics, 2012).

De acordo com Food and Agriculture Organization of the United Nations (2004), a bactéria C. botulinum encaixa-se na categoria $\mathrm{C}$ de identificação de perigos nas fórmulas infantis, ou seja, a causalidade é menos provável ou ainda não foi comprovada. Em 2001, um caso de botulismo infantil no Reino Unido foi possivelmente ligado à ingestão de fórmula infantil contaminada com esporos de C. botulinum. Na busca por respostas, dois laboratórios diferentes analisaram a amostra com técnicas genéticas distintas e identificaram estirpes variadas de $C$. botulinum nas fezes da criança, assim como em uma lata de fórmula em uso no momento da manifestação da doença e em uma lata fechada proveniente do mesmo lote. Como resultado, observou-se que uma mesma estirpe foi identificada na fórmula aberta e nas fezes da criança; no entanto, a mesma não foi encontrada na lata lacrada, indicando que a fonte de infecção não necessariamente foi a fórmula (Brett et al., 2005; Johnson et al., 2005).

Um estudo coordenado por Barash et al. (2010) utilizou uma base de dados contendo casos de crianças diagnosticadas com botulismo infantil que faziam uso de fórmula por pelo menos quatro semanas antes do episódio da doença. Foram investigadas 39 fórmulas - 30 provenientes do domicílio das crianças diagnosticadas e nove obtidas de supermercados. Após avaliação dos dados, constatou-se que $31 \%$ das amostras continham esporos de 12 espécies diferentes de Clostridium, no entanto, nenhuma delas era pertencente a espécies potencialmente causadoras de botulismo infantil, não sendo possível mais uma vez comprovar a responsabilidade do micro-organismo como causa direta da doença.

Um caso de botulismo infantil foi registrado na França acometendo um lactente de 11 semanas alimentado exclusivamente de leite materno, com exceção de uma única mamadeira de fórmula. $\mathrm{O}$ diagnóstico foi confirmado com o isolamento de C. botulinum tipo B e de toxina botulínica tipo B. Foram realizados testes da poeira ambiental da residência e o resultado foi negativo, no entanto o leite materno e o artificial não foram testados (Bernardor et al., 2018). 


\subsubsection{Klebsiella pneumoniae}

A Klebsiella pneumoniae (K. pneumoniae) é responsável por infecções hospitalares, como pneumonias, infecções teciduais, septicemias e infecções de trato urinário, que atingem com maior severidade indivíduos com o sistema imune debilitado, como os lactentes, possuindo morbimortalidade severa (AlcantarCuriel et al., 2004; Casolari et al., 2005). Após a bactéria ser ingerida e sobreviver ao ambiente estomacal, esta é capaz de se proteger contra as células de defesa imunológicas, colonizar o trato intestinal e migrar para outros tecidos, invadindo células e dando início a um processo infeccioso, principalmente no trato urinário (Struve et al., 2008). O controle desse micro-organismo é dificultado por sua habilidade de ser resistente a antibióticos e produzir fatores capazes de neutralizar ou reduzir a ação de células do sistema imune (Struve et al., 2008; Zhou et al., 2011).

A alimentação artificial pode ter associação com a colonização de bactérias resistentes como a K. pneumoniae no cólon de recém-nascidos (Vieira et al., 1999). Em 2004, a K. pneumoniae foi classificada na categoria "B" de perigos em fórmulas infantis em pó, ou seja, esses micro-organismos são causadores de doenças e foram encontrados em fórmulas infantis, no entanto seu vínculo como veículo da infecção alimentar não foi comprovado (Food and Agriculture Organization of the United Nations, 2004). Muytjens et al. (1988) analisaram 141 fórmulas infantis, sendo a K. pneumoniae identificada em 13 amostras. Outro estudo realizado por Carneiro et al. (2003) avaliou um total de 90 amostras de fórmulas coletadas do lactário do Hospital Universitário Pedro Ernesto, no Rio de Janeiro (HUPE/UERJ). Do total, 23 amostras estavam contaminadas por diferentes bactérias, sendo que as predominantes foram $K$. pneumoniae, K. planticol, Citrobacter freundii e Cedacea davisae (Carneiro et al., 2003).

Um trabalho realizado por Zhou et al. (2011) analisou 30 amostras de fórmulas infantis adquiridas de supermercados na China para identificação de bactérias e teste de resistência a antibióticos. Das amostras totais, a K. pneumoniae pôde ser isolada em seis, sendo $71,4 \%$ resistentes a seis ou mais tipos diferentes de antibióticos, o que reforça a importância no controle dos patógenos para a segurança dos alimentos destinados ao público infantil.

\subsubsection{Staphylococcus aureus}

Algumas espécies de Staphylococcus são capazes de produzir exotoxinas - enzimas ou proteínas - como as enterotoxinas provenientes de sua multiplicação e metabolismo, sendo estas posteriormente disponibilizadas na circulação sanguínea do hospedeiro (Tortora et al., 2002). A ingestão de toxinas pré-elaboradas nos alimentos é capaz de suprimir a resposta imune pelo estímulo de resposta inespecífica de células de defesa e liberar um volume grande de citocinas, instaurando um quadro de intoxicação sistêmica no organismo (Marrack \& Kappler, 1990).

Esses micro-organismos também possuem em sua parede celular proteínas que, ao interagir com as células do hospedeiro, são capazes de desenvolver fatores de virulência em infecções (Palmqvist et al., 2002). Além disso, algumas proteínas extracelulares contribuem para sua instalação e subsequente dano ao organismo, sendo capazes de aderir à parede celular auxiliando a bactéria na neutralização das células de defesa (Vojtov et al., 2002). Os sintomas mais comuns da toxicidade por Staphylococcus são diarreia, cólica abdominal, náusea e vômito (Le Loir et al., 2003).

A espécie Staphylococcus aureus (S. aureus) é comumente detectada na pele e na mão de manipuladores de alimentos, sendo assim uma fonte importante de contaminação direta e/ou indireta com a ingestão de produtos por eles manipulados (Tortora et al., 2017). Em 1980, Singh et al. analisaram 10 amostras de alimentos infantis enlatados em pó, sendo sete fórmulas infantis e três produtos alimentícios infantis à base de leite e cereais, todos adquiridos de supermercados. Da quantidade total, nove marcas estavam altamente contaminadas, o que indica possíveis falhas durante o processamento ou mesmo na distribuição ou na reconstituição do produto (Singh et al., 1980). 
No ano de 2004, um grande surto ligado à ingestão de $S$. aureus foi relatado na China, em que mais de 150 crianças foram hospitalizadas apresentando sintomas como febre, diarreia e vômito, após o consumo de fórmula láctea contaminada (Dairy Reporter, 2004). Outro estudo realizado na China, por Wang et al. (2010) analisou 367 amostras de alimentos infantis obtidos de 80 mercearias, sendo 143 fórmulas. Do total, 30 amostras estavam contaminadas por S. aureus, sendo 11,2\% (16) delas de leite em pó (Wang et al., 2012).

\subsubsection{Bacillus cereus}

O Bacillus cereus (B. cereus) pode causar dois tipos de doenças alimentares, sendo estas as síndromes diarreicas e eméticas (Bhunia, 2008). Na síndrome diarreica, há ingestão de alimentos contaminados por bactérias que, ao colonizar e se multiplicar no intestino delgado, produzem enterotoxinas capazes de formar poros na parede celular, ocasionando perda de íons e água (Arnesen et al., 2008). Já as síndromes eméticas são causadas pelo consumo de alimentos contaminados por toxinas - cereulidas - pré-formadas durante o armazenamento do produto ou da matéria-prima (Arnesen et al., 2008). A toxina pode causar sintomas como vômitos por se ligar a receptores do nervo vago no estômago, além de insuficiência hepática pela degeneração dos hepatócitos e pela intoxicação (Bhunia, 2008).

Produtos lácteos em pó são frequentemente contaminados com B. cereus e seus esporos (Reyes et al., 2007). Os esporos não são capazes de germinar em alimentos com baixa atividade de água, no entanto, quando os produtos são reconstituídos e mantidos em temperatura ambiente, esporos viáveis podem germinar e começar a produzir toxinas, podendo esse processo ser continuado sob refrigeração (Jaquette \& Beuchat, 1998). Portanto, justifica-se a preocupação da veiculação desses micro-organismos em fórmulas infantis. Um estudo realizado por Reyes et al. (2007) avaliou 381 amostras de alimentos em pó à base de leite oferecidos por um programa de alimentação escolar no Chile. Do total, 175 amostras (45,9\%) estavam contaminadas por esporos de B. cereus.

Organji et al. (2015) analisaram 110 amostras de alimentos destinados a lactentes e crianças de primeira infância à base de arroz e leite, dentre elas 20 fórmulas infantis e fezes de crianças com idades entre oito dias e dois anos apresentando quadro de diarreia na Arábia Saudita e Egito entre 2012 e 2013. Dentre as fórmulas, 12 amostras estavam contaminadas por bactérias do gênero Bacillus, sendo quatro $(33,3 \%)$ delas confirmadas como B. cereus. A presença desse micro-organismo também foi relacionada a $62,6 \%$ dos casos de diarreia nos lactentes e crianças participantes da pesquisa. Estudo similar foi desenvolvido por Di Pinto et al. (2013) entre 2008 e 2009, na Itália, onde foram analisadas 60 amostras de fórmulas infantis em pó, constatando-se a contaminação de 11 delas com bactérias do gênero Bacillus $\left(<10^{6} \mathrm{UFC} / \mathrm{g}\right)$, sendo cinco identificadas como B. cereus.

Um estudo realizado na Suíça buscou analisar alimentos destinados ao público infantil como purês de batata em pó e fórmulas infantis comercializados em supermercados. Como resultado, $78 \%$ das fórmulas estavam contaminadas por B. cereus sensu lato (B. cereus s. l.) (Heini et al., 2018). Recentes trabalhos realizados na China também buscaram identificar a presença de $B$. cereus em fórmulas infantis e/ou fórmulas infantis para lactentes. Zhang et al. (2017), Xin et al. (2018) e Pei et al. (2018) constataram contaminações de $8,2 \%, 36,1 \%$ e $100 \%$, respectivamente, em suas pesquisas. A possível contaminação por $B$. cereus também foi testada em uma planta de produção de fórmulas infantis de leite de cabra em 2016 na China. Foram coletadas 970 amostras de três plantas diferentes contemplando o ambiente de produção, os funcionários (vestimenta e mãos), o material de transporte e as fórmulas em várias etapas de produção. Do total, 36,1\% obtiveram resultado positivo para B. cereus s. l., sendo a bactéria detectada em 7 das 15 amostras coletadas do produto final pronto para comercialização (Liu et al., 2018).

Mesmo que a contaminação venha a ocorrer em níveis baixos, esta torna-se uma grande preocupação para a saúde de crianças, pois a matriz alimentar das fórmulas propicia o crescimento de bactérias e a produção de esporos, ou seja, esses alimentos em pó podem vir a ser veículos de infecções alimentares (Reyes et al., 2007). Portanto, é necessário um monitoramento contínuo e intensivo da presença de micro-organismos, 
assim como esporos e toxinas nos alimentos na busca da garantia de segurança e saúde para infantes e crianças consumidores de fórmulas (Di Pinto et al., 2013).

\section{Conclusões}

Concluiu-se que os micro-organismos mais frequentemente relacionados com surtos alimentares em lactentes e crianças de primeira infância foram Salmonella enterica e Cronobacter sakazakii. Para garantia da segurança microbiológica das fórmulas lácteas "de partida”, para lactentes e de transição, são necessários cuidados desde a sua produção industrial como a implementação de medidas de Boas Práticas de Fabricação, a Análise dos Pontos Críticos de Controle e o treinamento higiênico-sanitário para manipuladores, até o preparo e a administração em nível domiciliar ou hospitalar com a correta manipulação e procedimentos sanitários adequados.

\section{Referências}

Alcantar-Curiel, D., Carlos Tinoco, J., Gayosso, C., Carlos, A., Daza, C., Perez-Prado, M. C., Salcido, L., Santos, J. I., \& Alpuche-Aranda, C. M. (2004). Nosocomial bacteremia and urinary tract infections caused by extended-spectrum $\beta$-Lactamase: Producing Klebsiella pneumoniae with plasmids carrying both SHV-5 and TLA-1 genes. Clinical Infectious Diseases, 38(8), 1067-1074. PMid:15095209. http://dx.doi.org/10.1086/382354

American Academy of Pediatrics - AAP. (2012). Summaries of infectious diseases. In L. K. Pickering, C. J. Baker, D. W. Kimberlin \& S. S. Long (Eds.), Red Book: 2012 report of the committee on infectious diseases (Section 3, pp. 219-797). Elk Grove Village: American Academy of Pediatrics.

Arnesen, L. P. S., Fagerlund, A., \& Granum, P. E. (2008). From soil to gut: Bacillus cereus and its food poisoning toxins. FEMS Microbiology Reviews, 32(4), 579-606. PMid:18422617. http://dx.doi.org/10.1111/j.1574-6976.2008.00112.x

Barash, J. R., Hsia, J. K., \& Arnon, S. S. (2010). Presence of soil-dwelling clostridia in commercial powdered infant formulas. The Journal of Pediatrics, 156(3), 402-408. PMid:20004414. http://dx.doi.org/10.1016/j.jpeds.2009.09.072

Bar-Oz, B., Preminger, A., Peleg, O., Block, C., \& Arad, I. (2001). Enterobacter sakazakii infection in the newborn. Acta Paediatrica, 90(3), 356-358. PMid:11332182. http://dx.doi.org/10.1080/080352501300067857

Bernardor, J., Neveu, J., Haas, H., Pitelet, G., Popoff, M.-R., Mazuet, C., Bérard, E., Boulay, C., \& Chabrol, B. (2018). Infant botulism: Two case reports and electroneuromyogram findings. Archives de Pediatrie, 25(5), 340-343. PMid:29887515. http://dx.doi.org/10.1016/j.arcped.2018.05.002

Beuchat, L. R., Komitopoulou, E., Beckers, H., Betts, R. P., Bourdichon, F., Fanning, S., Joosten, H. M., \& Ter Kuile, B. H. (2013). Low-water activity foods: increased concern as vehicles of foodborne pathogens. Journal of Food Protection, 76(1), 150172. PMid:23317872. http://dx.doi.org/10.4315/0362-028X.JFP-12-211

Bhunia, A. K. (2008). Bacillus cereus and Bacillus anthracis. In A. K. Bhunia (Ed.), Foodborne microbial pathogens: Mechanisms and pathogenesis (Cap. 7, pp. 135-148). West Lafayette: Springer.

Bhutta, Z. A., Ahmed, T., Black, R. E., Cousens, S., Dewey, K., Giugliani, E., Haider, B. A., Kirkwood, B., Morris, S. S., Sachdev, H. P. S., \& Shekar, M. (2008). What works? Interventions for maternal and child undernutrition and survival. Lancet, 371(9610), 417-440. PMid:18206226. http://dx.doi.org/10.1016/S0140-6736(07)61693-6

Biering, G., Karlsson, S., Clark, N. C., Jônsdôttir, K. E., Ludvigsson, P., \& Steingrimsson, O. (1989). Three cases of neonatal meningitis caused by Enterobacter sakazakii in powdered milk. Journal of Clinical Microbiology, 27(9), 2054-2056. PMid:2778070.

Brandão, M. L. L., Umeda, N. S., \& Filippis, I. (2018). Cronobacter spp.: Infecções, ocorrência e regulação em alimentos: Uma revisão no Brasil. Brazilian Journal of Food Technology, 21(e2017074), 1-9.

Brasil. Agência Nacional de Vigilância Sanitária - ANVISA. (2001). Resolução-RDC no 12, de 2 de janeiro de 2001. Regulamento técnico sobre padrões microbiológicos para alimentos. Diário Oficial [da] República Federativa do Brasil, Brasília. Recuperado em 20 de novembro de 2017, de

http://portal.anvisa.gov.br/documents/33880/2568070/RDC_12_2001.pdf/15ffddf6-3767-4527-bfac-740a0400829b

Brasil. Agência Nacional de Vigilância Sanitária - ANVISA. (2014). Perguntas e respostas sobre fórmulas infantis. Brasília: Gerência de Produtos Especiais, Gerência Geral de Alimentos.

Brett, M. M., Mclauchlin, J., Harris, A., O’Brien, S., Black, N., Forsyth, R. J., Roberts, D., \& Bolton, F. J. (2005). A case of infant botulism with a possible link to infant formula milk powder: evidence for the presence of more than one strain of Clostridium botulinum in clinical specimens and food. Journal of Medical Microbiology, 54(8), 769-776. PMid:16014431. http://dx.doi.org/10.1099/jmm.0.46000-0

Brook, I. (2007). Infant botulism. Journal of Perinatology, 27(3), 175-180. PMid:17314986. http://dx.doi.org/10.1038/sj.jp.7211651

Brouard, C., Espié, E., Weill, F.-X., Kérouanton, A., Brisabois, A., Forgue, A.-M., Vaillant, V., \& de Valk, H. (2007). Two consecutive large outbreaks of Salmonella enterica Serotype Agona infections in infants linked to the consumption of powdered 
infant formula. The Pediatric Infectious Disease Journal, 26(2), 148-152. PMid:17259878. http://dx.doi.org/10.1097/01.inf.0000253219.06258.23

Bula-Rudas, F. J., Rathore, M. H., \& Maraqa, N. F. (2015). Salmonella infections in childhood. Advances in Pediatrics, 62(1), 29-58. PMid:26205108. http://dx.doi.org/10.1016/j.yapd.2015.04.005

Cahill, S. M., Wachsmuth, I. K., Costarrica, M. L., \& Embarek, P. K. B. (2008). Powdered Infant formula as a source of Salmonella infection in infants. Food Safety, 46(2), 268-273. PMid:18171262.

Carneiro, L. A. M., Silva, A. P. S., Merquior, V. L. C., \& Queiroz, M. L. P. (2003). Antimicrobial resistance in Gram-negative bacilli isolated from infant formulas. FEMS Microbiology Letters, 228(2), 175-179. PMid:14638421. http://dx.doi.org/10.1016/S0378-1097(03)00739-0

Casolari, C., Pecorari, M., Fabio, G., Cattani, S., Venturelli, C., Piccinini, L., Tamassia, M. G., Gennari, W., Sabbatini, A. M. T., Leporati, G., Marchegiano, P., Rumpianesi, F., \& Ferrari, F. (2005). A simultaneous outbreak of Serratia marcescens and Klebsiella pneumoniae in a neonatal intensive care unit. The Journal of Hospital Infection, 61(4), 312-320. PMid:16198443. http://dx.doi.org/10.1016/j.jhin.2005.03.005

Centers for Disease Control and Prevention - CDC. (1993). Salmonella serrotype Tennessee in powdered milk products and infant formula: Canada and United States, 1993. Journal of the American Medical Association, 270(4), 432. PMid:8320774. http://dx.doi.org/10.1001/jama.1993.03510040036011

Centers for Disease Control and Prevention - CDC. (2002). Enterobacter sakazakii infections associated with the use of powdered infant formula: Tennessee, 2001. Morbidity and Mortality Weekly Report, 51(14), 297-300. PMid:12002167.

Collins, R. N., Treger, M. D., Goldsby, J. B., Boring Junior, R. D., Coohon, D. B., \& Barr, R. N. (1968). Interstate outbreak of Salmonella newbrunswick infection traced to powdered milk. Journal of the American Medical Association, 203(10), 838-844. PMid:5694205. http://dx.doi.org/10.1001/jama.1968.03140100020005

Cunha, A. J. L. A., Leite, A. J. M., \& Almeida, I. S. (2015). The pediatrician's role in the first thousand days of the child: The pursuit of healthy nutrition and development. Jornal de Pediatria, 91(6, Supl.1), S44-S51. PMid:26351769. http://dx.doi.org/10.1016/j.jped.2015.07.002

Dairy Reporter. (2004). Baby milk poisoning. Recuperado em 6 de dezembro de 2017, de http://www.dairyreporter.com/Markets/Baby-milk-poisoning

Di Pinto, A., Bonerba, E., Bozzo, G., Ceci, E., Terio, V., \& Tantillo, G. (2013). Occurence of potentially enterotoxigenic Bacillus cereus in infant milk powder. European Food Research and Technology, 237(2), 275-279. http://dx.doi.org/10.1007/s00217-013$1988-8$

Fei, P., Jiang, Y., Jiang, Y., Yuan, X., Yang, T., Chen, J., Wang, Z., Kang, H., \& Forsythe, S. J. (2017). Prevalence, molecular characterization, and antibiotic susceptibility of Cronobacter sakazakii isolates from powdered infant formula collected from chinese retail markets. Frontiers in Microbiology, 8, 2026. PMid:29089940. http://dx.doi.org/10.3389/fmicb.2017.02026

Fiore, A., Casale, M., \& Aureli, P. (2008). Enterobacter sakazakii: Epidemiology, clinical presentation, prevention and control. Annali dell'Istituto Superiore di Sanita, 44(3), 275-280. PMid:18946167.

Food and Agriculture Organization of the United Nations - FAO. World Health Organization - WHO. (2004). Expert meeting on Enterobacter sakazakii and other microorganisms in powdered infant formula: Meeting report (Microbiological Risk Assessment Series, No. 6). Geneva: WHO.

Food and Agriculture Organization of the United Nations - FAO. World Health Organization - WHO. (2006). Technical meeting on Enterobacter sakazakii and Salmonella in powdered infant formula: Meeting report (Microbiological Risk Assessment Series, No. 10). Geneva: WHO.

Food and Agriculture Organization of the United Nations - FAO. World Health Organization - WHO. Codex Alimentarius. (2009). CAC/RCP 66-2008: Code of hygienic practice for powdered formulae for infants and young children. Rome: FAO.

Haimovich, B., \& Venkatesan, M. M. (2006). Shigella and Salmonella: Death as a means of survival. Microbes and Infection, 8(2), 568-577. PMid:16297650. http://dx.doi.org/10.1016/j.micinf.2005.08.002

Heini, N., Stephan, R., Ehling-Schulz, M., \& Johler, S. (2018). Characterization of Bacillus cereus group isolates from powdered food products. International Journal of Food Microbiology, 283, 59-64. PMid:30099996.

http://dx.doi.org/10.1016/j.ijfoodmicro.2018.06.019

International Commision on Microbial Specifications for Foods - ICMSF. (2015). Microrganismos em alimentos. São Paulo: Blucher.

Iversen, C., \& Forsythe, S. (2003). Risk profile of Enterobacter sakazakii, an emergent pathogen associated with infant milk formula. Trends in Food Science \& Technology, 14(11), 443-454. http://dx.doi.org/10.1016/S0924-2244(03)00155-9

Jaquette, C. B., \& Beuchat, L. R. (1998). Survival and growth of psychrotrophic Bacillus cereus in dry and reconstituted infant rice cereal. Journal of Food Protection, 61(12), 1629-1635. PMid:9874340. http://dx.doi.org/10.4315/0362-028X-61.12.1629

Johnson, E. A., Tepp, W. H., Bradshaw, M., Gilbert, R. J., Cook, P. E., \& Mcintosh, E. D. G. (2005). Characterization of Clostridium botulinum strains associated with an infant botulism case in the United Kingdom. Journal of Clinical Microbiology, 43(6), 2602-2607. PMid:15956371. http://dx.doi.org/10.1128/JCM.43.6.2602-2607.2005

Jourdan, N., Le Hello, S., Delmas, G., Clouzeau, J., Manteau, C., Désaubliaux, B., Chagnon, V., Thierry-Bled, F., Demare, N., Weill, F. X., \& De Valk, H. (2008). Nationwide outbreak of Salmonella enterica serotype give infections in infants in France, linked to infant milk formula, September 2008. Eurosurveillance, 13(39). PMid:18822245. 
Kim, S. A., Oh, S. W., Lee, Y. M., Imm, J. Y., Hwang, I. G., Kang, D. H., \& Rhee, M. S. (2011). Microbial contamination of food products consumed by infants and babies in Korea. Letters in Applied Microbiology, 53(5), 532-538. PMid:21883321. http://dx.doi.org/10.1111/j.1472-765X.2011.03142.x

Le Loir, Y., Baron, F., \& Gautier, M. (2003). Staphylococcus aureus and food poisoning. Genetics and Molecular Research, 2(1), 63-76. PMid:12917803.

Lehner, A., Riedel, K., Eberl, L., Breeuwer, P., Diep, B., \& Stephan, R. (2005). Biofilm formation, extracellular polysaccharide production, and cell-to-cell signaling in various Enterobacter sakazakii strains: Aspects promoting environmental persistence. Journal of Food Protection, 68(11), 2287-2294. PMid:16300064. http://dx.doi.org/10.4315/0362-028X-68.11.2287

Liu, Y., Ge, W., Zhang, J., Li, X., Wu, X., Li, T., Zhang, X., \& Wang, X. (2018). Detection of Bacillus cereus sensu lato from environments associated with goat milk powdered infant formula production facilities. International Dairy Journal, 83, 10-16. http://dx.doi.org/10.1016/j.idairyj.2018.02.005

Machado, T. F. (2013). Patógenos emergentes em alimentos. Fortaleza: Embrapa Agroindústria Tropical.

Mardaneh, J., \& Dallal, M. M. S. (2017). Study of Cronobacter sakazakii strains isolated from powdered milk infant formula by phenotypic and molecular methods in Iran. Archives of Pediatric Infectious Diseases, 5(1), e38867.

Marrack, P., \& Kappler, J. (1990). The staphylococcal enterotoxins and their relatives. Science, 248(4956), 705-711. PMid:2185544. http://dx.doi.org/10.1126/science.2185544

Morlay, A., Piat, F., Mercey, T., \& Roupioz, Y. (2016). Immunological detection of Cronobacter and Salmonella in powdered infant formula by plasmonic label-free assay. Letters in Applied Microbiology, 62(6), 459-465. PMid:27005955. http://dx.doi.org/10.1111/lam.12570

Mozetic, R. M., Silva, S. D. C., \& Ganen, A. P. (2016). A importância da nutrição nos primeiros mil dias. Revista Eletrônica Acervo Saúde, 8(2), 876-884.

Muytjens, H. L., Roelofs-Willemse, H., \& Jaspar, G. H. J. (1988). Quality of powdered substitutes for breast milk with regard to members of the family Enterobacteriaceae. Journal of Clinical Microbiology, 26(4), 743-746. PMid:3284901.

Nazarowec-White, M., \& Farber, J. M. (1997). Incidence, survival, and growth of Enterobacter sakazakii in infant formula. Journal of Food Protection, 60(3), 226-230. http://dx.doi.org/10.4315/0362-028X-60.3.226

Ohl, M. E., \& Miller, S. I. (2001). Salmonella: A model for bacterial pathogenesis. Annual Review of Medicine, 52(1), $259-274$. PMid:11160778. http://dx.doi.org/10.1146/annurev.med.52.1.259

Organji, S. R., Abulreesh, H. H., Elbanna, K., Osman, G. E. H., \& Khider, M. (2015). Occurrence and characterization of toxigenic Bacillus cereus in food and infant feces. Asian Pacific Journal of Tropical Biomedicine, 5(7), 515-520. http://dx.doi.org/10.1016/j.apjtb.2015.04.004

Orvay, J. A. C., Hervás, A., Hurtado, A., \& Bonet, B. (2013). Meningitis por Salmonella tras toxiinfección alimentaria en lactante alimentado con lactancia artificial. Anales de Pediatria, 79(4), 205-274. PMid:23838286.

Palmqvist, N., Foster, T., Tarkowski, A., \& Josefsson, E. (2002). Protein A is a virulence factor in Staphylococcus aureus arthritis and septic death. Microbial Pathogenesis, 33(5), 239-249. PMid:12473438. http://dx.doi.org/10.1006/mpat.2002.0533

Park, J. K., Seok, W. S., Choi, B. J., Kim, H. M., Lim, B. K., Yoon, S. S., Kim, S., Kim, Y. S., \& Park, J. Y. (2004). Salmonella enterica serovar London infections associated with consumption of infant formula. Yonsei Medical Journal, 45(1), 43-48. PMid:15004867. http://dx.doi.org/10.3349/ymj.2004.45.1.43

Parra-Flores, J., Cerda-Leal, F., Contreras, A., Valenzuela-Riffo, N., Rodríguez, A., \& Aguirre, J. (2018). Cronobacter sakazakii and microbiological parameters in dairy formulas associated with a food alert in Chile. Frontiers in Microbiology, 9, 1708. PMid:30108565. http://dx.doi.org/10.3389/fmicb.2018.01708

Pei, X., Yang, S., Zhan, L., Zhu, J., Song, X., Hu, X., Liu, G., Ma, G., Li, N., \& Yang, D. (2018). Prevalence of Bacillus cereus in powdered infant and powdered follow-up formula in China. Food Control, 93, 101-105. http://dx.doi.org/10.1016/j.foodcont.2018.05.049

Reyes, J. E., Bastías, J. M., Gutiérrez, M. R., \& Rodríguez, M. O. (2007). Prevalence of Bacillus cereus in dried milk products used by Chilean School Feeding Program. Food Microbiology, 24(1), 1-6. PMid:16943088. http://dx.doi.org/10.1016/j.fm.2006.04.004

Rosow, L. K., \& Strober, J. B. (2015). Infant botulism: review and clinical update. Pediatric Neurology, 52(5), 487-492. PMid:25882077. http://dx.doi.org/10.1016/j.pediatrneurol.2015.01.006

Rossi, P. (2007). Avaliação de perigos microbiológicos no preparo de fórmulas infantis em lactário hospitalar (Dissertação de mestrado). Faculdade de Engenharia de Alimentos, Universidade Estadual de Campinas, Campinas.

Rowe, B., Hutchinson, D. N., Gilbert, R. J., Hales, B. H., Begg, N. T., Dawkins, H. C., Jacob, M., Rae, F. A., \& Jepson, M. (1987). Salmonella ealing infections associated with consumption of infant dried milk. Lancet, 2(330), 900-903. PMid:2889093. http://dx.doi.org/10.1016/S0140-6736(87)91384-5

Sá, P. B. Z. R., Alves, A. T. S., Oliveira, M. N., Bogsan, C. S., Guimarães, J. T., Pimentel, T. C., Cruz, A. G., \& Antunes, A. E. C. (2017). Leites infantis. In A. G. Cruz, P. B. Zacarchenco, C. A. F. Oliveira \& C. H. Corassin (Eds.), Processamento de leites de consumo (Cap. 8, pp. 195-217). Rio de Janeiro: Elsevier.

Shinohara, N. K. S., Barros, V. B., Jimenez, S. M. C., Machado, E. C. L., Dutra, R. A. F., \& Lima Filho, J. L. (2008). Salmonella spp., importante agente patogênico veiculado em alimentos. Ciência \& Saúde Coletiva, 13(5), 1669-1674. PMid:18813667. http://dx.doi.org/10.1590/S1413-81232008000500031 
Simmons, B. P., Gelfand, M. S., Haas, M., Metts, L., \& Ferguson, J. (1989). Enterobacter sakazakii infections in neonates associated with intrinsic contamination of a powdered infant formula. Infection Control and Hospital Epidemiology, 10(9), 398401. PMid:2794464. http://dx.doi.org/10.2307/30144207

Singh, R. S., Singh, S., Batish, V. K., \& Ranganathan, B. (1980). Bacteriological quality of infant milk foods. Journal of Food Protection, 43(5), 340-342. PMid:30822869. http://dx.doi.org/10.4315/0362-028X-43.5.340

Soler, P., Herrera, S., Rodríguez, J., Cascante, J., Cabral, R., Echeita-Sarriondia, A., \& Mateo, S. (2008). Nationwide outbreak of Salmonella enterica serotype Kedougou infection in infants linked to infant formula milk, Spain, 2008. Eurosurveillance, 13(35). PMid:18761885. http://dx.doi.org/10.2807/ese.13.35.18963-en

Struve, C., Bojer, M., \& Krogfelt, K. A. (2008). Characterization of Klebsiella pneumoniae Type 1 Fimbriae by detection of phase variation during colonization and infection and impact on virulence. Infection and Immunity, 76(9), 4055-4065. PMid:18559432. http://dx.doi.org/10.1128/IAI.00494-08

Threlfall, E. J., Ward, L. R., Hampton, M. D., Ridley, A. M., Rowe, B., Roberts, D., Gilbert, R. J., Van Someren, P., Wall, P. G., \& Grimont, P. (1998). Molecular fingerprinting defines a strain of Salmonella enterica serotype Anatum responsible for an international outbreak associated with formula-dried milk. Epidemiology and Infection, 121(2), 289-293. PMid:9825779. http://dx.doi.org/10.1017/S0950268898001149

Tortora, G. J., Funke, B. R., \& Case, C. L. (2002). Microbiologia (6. ed.). São Paulo: Artmed.

Tortora, G. J., Funke, B. R., \& Case, C. L. (2017). Doenças microbianas do sistema digestório. In G. J. Tortora, B. R. Funke \& C. L. Case (Eds.), Microbiologia (12. ed., Cap. 25, pp. 707-745). Porto Alegre: Artmed.

Usera, M. A., Echeita, A., Aladueña, A., Blanco, M. C., Reymundo, R., Prieto, M. I., Tello, O., Cano, R., Herrera, D., \& MartinezNavarro, F. (1996). Interregional foodborne salmonellosis outbreak due to powdered infant formula contaminated with lactosefermenting Salmonella virchow. European Journal of Epidemiology, 12(4), 377-381. PMid:8891542. http://dx.doi.org/10.1007/BF00145301

Van Acker, J., De Smet, F., Muyldermans, G. T., Bougatef, A., Naessens, A., \& Lauwers, S. (2001). Outbreak of necrotizing enterocolitis associated with Enterobacter sakazakii in powdered milk formula. Journal of Clinical Microbiology, 39(1), 293-297. PMid:11136786. http://dx.doi.org/10.1128/JCM.39.1.293-297.2001

Vieira, L. A., Castro, E. A. R., Duarte, J. L. B., Pinheiro, S. R., Suassuna, Í., \& Pereira, J. A. A. (1999). Colonização intestinal de recém-natos por enterobactérias multirresistentes a antimicrobianos em unidade neonatal. Jornal de Pediatria, 75(2), 83-90. PMid:14685546. http://dx.doi.org/10.2223/JPED.263

Vojtov, N., Ross, H. F., \& Novick, R. P. (2002). Global repression of exotoxin synthesis by staphylococcal superantigens. Proceedings of the National Academy of Sciences of the United States of America, 99(15), 10102-10107. PMid:12110733. http://dx.doi.org/10.1073/pnas.152152499

Wang, X., Meng, J., Zhang, J., Zhou, T., Zhang, Y., Yang, B., Xi, M., \& Xia, X. (2012). Characterization of Staphylococcus aureus isolated from powdered infant formula milk and infant rice cereal in China. International Journal of Food Microbiology, 153(1-2), 142-147. PMid:22119265. http://dx.doi.org/10.1016/j.ijfoodmicro.2011.10.030

Weir, E. (2002). Powdered infant formula and fatal infection with Enterobacter sakazakii. Canadian Medical Association Journal, 166(12), 1570. PMid:12074129.

Whitworth, J. J. (2017). Salmonella in infant formula sickens 20. Recuperado em 6 de dezembro de 2017, de https://www.foodqualitynews.com/Article/2017/12/04/Lactalis-issues-recall-after-link-to-Salmonellaoutbreak?utm_source=newsletter_daily\&utm_medium=email\&utm_campaign=04-Dec2017\&c=Hm0G rE2seJcFqGx7CNWM4Q\%3D\%3D\&p2

Willsher, K. (2018). Lactalis to withdraw $12 \mathrm{~m}$ boxes of baby milk in Salmonella scandal. Recuperado em 15 de janeiro de 2017, de https://www.theguardian.com/world/2018/jan/14/lactalis-baby-milk-salmonella-scandal-affects-83-countries-ceo-says

World Health Organization - WHO. Food and Agriculture Organization of the United Nations - FAO. (2007). Safe preparation, storage and handling of powdered infant formula: Guidelines. Geneva: WHO.

Wu, S., Jiang, Y., Lou, B., Feng, J., Zhou, Y., Guo, L., Forsythe, S. J., \& Man, C. (2018). Microbial community structure and distribution in the air of a powdered infant formula factory based on cultivation and high-throughput sequence methods. Journal of Dairy Science, 101(8), 6915-6926. PMid:29729917. http://dx.doi.org/10.3168/jds.2017-13968

Xin, G., Yinping, D., Shaofei, Y., Yujie, H., Fanning, S., Jiahui, W., \& Fengqin, L. (2018). Contamination and characterization of multiple pathogens in powdered formula at retail collected between 2014 and 2015 in China. Food Control, 87, 40-45. http://dx.doi.org/10.1016/j.foodcont.2017.12.014

Yang, B., Zhao, H., Cui, S., Wang, Y., Xia, X., Xi, M., Wang, X., Meng, J., \& Ge, W. (2014). Prevalence and characterization of Salmonella enterica in dried milk-related infant foods in Shaanxi, China. Journal of Dairy Science, 97(11), 6754-6760. PMid:25218754. http://dx.doi.org/10.3168/jds.2014-8292

Zhang, Y., Chen, J., Feng, C., Zhan, L., Zhang, J., Li, Y., Yang, Y., Chen, H., Zhang, Z., Zhang, Y., Mei, L., \& Li, H. (2017). Quantitative prevalence, phenotypic and genotypic characteristics of Bacillus cereus Isolated from retail infant foods in China. Foodborne Pathogens and Disease, 14(10), 564. PMid:28753035. http://dx.doi.org/10.1089/fpd.2017.2287

Zhou, X., Gao, J., Huang, Y., Fu, S., \& Chen, H. (2011). Antibiotic resistance pattern of Klebsiella pneumoniae and Enterobacter sakazakii isolates from powdered infant formula. African Journal of Microbiological Research, 5(19), 3073-3077. http://dx.doi.org/10.5897/AJMR10.867 